\title{
Simplified Block Adaptive Diversity Equalizer for Cellular Mobile Radio.*
}

\author{
Tugay Eyceöz and Alexandra Duel-Hallen \\ Center for Advanced Computing and Communication, \\ North Carolina State University, Box 7914, \\ Raleigh, NC 27695-7914 \\ E-mail: teyceoz@eos.ncsu.edu, sasha@eos.ncsu.edu
}

\begin{abstract}
We propose a reduced complexity antenna diversity combiner-equalizer receiver structure to combat multipath fading in Cellular Mobile Radio (CMR) communications. The technique utilizes block adaptation based on interpolated channel estimates and linear or decision feedback equalization. The receiver offers complexity reduction relative to previously proposed block adaptation methods without sacrificing performance.
\end{abstract}

${ }^{*}$ This research was supported by NSF grant NCR-9410227. 


\section{Introduction}

Performance of the CMR communication systems is significantly degraded by rapidly time-varying intersymbol interference (ISI) caused by multipath fading [1, 2]. Several diversity combiner-adaptive equalizer receiver structures were proposed to combat frequency selective fading. These structures feature multiple antennas, spaced sufficiently far apart so that their received signals fade independently, combined with equalizers used for mitigating the effects of ISI.

It is important to consider channel fading in the design of an adaptive equalizer. In CMR communications where the channel is fast fading, combiner equalizer parameters can be calculated periodically based on channel estimates or updated continuously using an adaptive equalization algorithm. We used the former approach. Specifically, block adaptation is used for our CMR communication system. In contrast to adaptive equalization, the receiver parameters in our system are computed periodically based on channel impulse response (CIR) estimates. The beginning of each data frame contains a known training sequence which is used to estimate CIR. However, for a fast fading channel, the CIR estimate obtained from training at the beginning of a data frame should be updated several times during the data frame. This time-varying CIR is determined by interpolating a set of estimated CIR values. Then the combiner-equalizer parameters are computed using interpolated channel estimates from each of the $L$ diversity channels, yielding the optimum performance in the MMSE sense.

In [3], Lo et al. used the same block-adaptive strategy. However, their receiver structure differs from ours. In their decision feedback receiver, they used an individual fractionally spaced feedforward filter for each diversity path and one common symbol spaced feedback filter. On the other hand, in [4], Balaban and Salz derived a symbol spaced Minimum Mean Square Error (MMSE) optimum receiver structure. They also incorporated diversity combining and adaptive equalization into a single receiver. Implementing this receiver requires knowledge of actual channel characteristics, since this receiver features a set of matched filters for each diversity branch. The output samples from the matched filters are summed and processed using a single symbol-spaced equalizer. We combined the block-adaptive strategy of [3] with the receiver structure similar to that in [4]. Our receiver has fractionally spaced matched filter for each diversity branch followed by a single symbol spaced Linear Equalizer 
(LE) or Decision Feedback Equalizer (DFE). This receiver structure has lower computational complexity than that of Lo et al., and does not require prior knowledge of channel parameters as that of Balaban and Salz.

In the following sections we describe our CMR communication model and discuss simulation results. We demonstrate that the proposed simplification does not result in performance degradation and compare the proposed receivers for various system parameters.

\section{CMR System Description}

A block diagram of the CMR communications system is presented in Figure 1(a). Our system specifications are compatible with the U.S. digital cellular system. A narrow-band time division multiple access (TDMA) and the $\pi / 4$-shifted DQPSK modulation method are used. The transmit and receive filters have square root raised cosine frequency response characteristics with a $35 \%$ rolloff factor. In this paper a discrete-time representation is used which corresponds to sampling all signals at $T / 2$, where $T$ is a symbol interval.

At the $l^{\text {th }}$ diversity branch, the received signal is

$$
\begin{aligned}
V_{l}(t) & =S(t) * h_{l}(t)+N_{l}(t) \\
& =\sum_{k} S(k T) h_{l}(t-k T)+N_{l}(t)
\end{aligned}
$$

where $S(k T)$ is the $\pi / 4$-shifted DQPSK data sequence at time $k T, N_{l}(t)$ is the AWGN with the variance $\sigma_{n}^{2}$, and $h_{l}(t)$ is the channel impulse response (CIR) which also includes the transmit and received filter responses.

For each diversity branch, the CMR channel is modeled in complex baseband representation as an FIR filter in the form of a T/2-spaced tapped-delay-line (TDL) as shown in Figure 1(b). The TDL consists of three complex tap coefficients, $c_{l}^{i}(k T) \mathrm{i}=1,2,3$, each representing a distinct multipath. The multipath power delay profile (MPDP) of the channel is specified with a three-paths "mountainous terrain" model with relative rms powers of $0,-5$, and $-15 \mathrm{~dB}[3,5]$. Each channel tap coefficient is an independent Rayleigh fading process simulated with the "modified Jakes model" $[6,7]$ using sixteen sinusoids with distinct Doppler frequencies up to a maximum Doppler frequency, $f_{d m}$ of $100 \mathrm{~Hz}$. Furthermore, complex additive white gaussian noise (AWGN) with variance $\sigma_{n}^{2}$ is added to the transmitted signal 
at the output of each diversity channel. The channel responses and the noise components are independent.

The signaling format of alternating training and data sequences within a long TDMA time slot is used. Each data frame consists of a training sequence of length $N_{t}$ symbols followed by an uncorrelated data sequence of length $N_{d}$ symbols. Therefore, the number of symbols in each transmitted frame is $\eta=N_{d}+N_{t}$. Since CIR estimates are updated at the beginning of each data frame, this type of periodic training improves the adaptation problem at the expense of decreased throughput,

$$
\% T=\frac{100 N_{d}}{N_{d}+N_{t}} \%=\frac{100 N_{d}}{\eta} \%
$$

To estimate CIR for each branch, block adaptation using a known training sequence is employed [3]. In our system, the overall CIR length, $N_{c}$, is fixed to six symbol periods of which five symbol periods are due to transmit and receive filter impulse responses, and one symbol period is due to the actual CIR of the CMR channel. The training sequence is 15 symbols long as suggested in [3].

A set of CIR estimates is obtained from the Block Least Squares (BLS) solution described in [3] during training intervals. The CIR estimates are updated during data transmission by interpolating a set of estimated CIR values [3]. The relative positions of the estimated and interpolated CIR samples within the TDMA time slot are illustrated in Fig 1(c). The normalized sampling rate of the CIR estimates satisfying Nyquist's sampling criterion, is defined as $[3]$

$$
\bar{f}=\frac{f_{s}}{2 f_{d m}}=\frac{1}{2 f_{d m} \eta T} \geq 1
$$

where $f_{s}$ is sampling rate. For example, for the maximum Doppler frequency of $f_{d m}=100 \mathrm{~Hz}$ and symbol rate of $\frac{1}{T}=24 \mathrm{ksps}$, the required frame length should be $\eta \leq 120$ symbols.

This block adaptation strategy was previously published [3], but its utilization in the receiver structure is new as described below.

We used interpolated CIR estimates, $\hat{\boldsymbol{h}}_{\boldsymbol{l}}$, from each of the L diversity channels to compute the receiver parameters yielding the optimum performance in the MMSE sense. The optimal receivers are derived under the assumption that the channel response $h_{l}(t)$ can be estimated 
perfectly, i.e., $\hat{h}_{l}(t)$ is given by the actual response $h_{l}(t)$. It was shown in [4] that first step in the implementation of the optimum linear or decision feedback equalizer is to pass the received signal $V_{l}(t)$ in (1) through a matched filter associated with the $l^{\text {th }}$ diversity channel. The outputs of the matched filters are then summed and processed using a symbol-spaced decision structure. The optimal configurations are depicted in Figures $2(\mathrm{a})$ and $2(\mathrm{~b})$ for the linear and the decision feedback for equalizers, respectively. Moreover, the tap coefficients of these equalizers are derived as in [4]. In [4], known channel responses are assumed. However, in the adaptive implementation, channel responses need to be estimated. In our implementation, the filter tap coefficients are determined by the current channel estimates obtained from block adaptation and interpolation as described above. The matched filters are also based on these CIR estimates. We implemented these matched filters as fractionally spaced (specifically $T / 2$ spaced), transversal filters as shown in Figure 2(c). The overall length of the matched filter is six symbol periods which is the length of the CIR estimate.

The computational complexity reduction of the new structure results from employing a single equalizer following a diversity combiner. Previously proposed adaptive receivers featured separate fractionally spaced equalizers associated with each diversity branch. Calculation of the equalizer coefficients is significantly simplified. The computational complexity is independent of the number of antenna elements as opposed to linear dependence in previous implementations $[3,8,9]$.

\section{Simulation Results}

Numerical results illustrate several performance characteristics of the proposed receivers. The system performance improves with increasing normalized sampling rate, $\bar{f}$, and diversity order, $L$. For all diversity cases, poor performance at the Nyquist sampling rate (i.e., $\bar{f}=1$ ) is observed [8]. This is illustrated in Fig. 3 for $L=1$. Near the minimum sampling rate, the Doppler spectral images of the CIR estimates are very close together. Hence, this high BER is caused by the severe aliasing distortion due to the interpolation filter which is not an ideal lowpass filter [3]. The performance improvement with increasing $\bar{f}$ results in an overhead of reduced system throughput, \%T (2). Our results for various Doppler frequencies and the number of diversity channels show that a value of $\bar{f}=2.0$ (i.e., twice the Nyquist sampling rate), with an associated $\% T=75 \%$ is a reasonable design choice for our CMR 
communications system (Fig. 3) [8].

Experiments in [8] and results reported in [3] show that the linear equalizer with 5 taps and decision feedback equalizer with 3 forward and 2 feedback taps are reasonable choices for the diversity combining equalization structures. We refer to these as the optimum diversity combiner linear equalizer (ODCLE $(5,0)$ ) and the optimum diversity combiner decision feedback equalizer (ODCDFE(3,2)) and use them in our simulations.

In [3], Lo et al. used a different equalization structure. Instead of utilizing matched filters, they used an individual fractionally spaced feedforward filter for each diversity path and one common symbol spaced feedback filter. The authors of [3] solved matrix equations with the order of $\left(L * N_{f}+N_{b}\right) \times\left(L * N_{f}+N_{b}\right)$ to calculate their DFE equalizer coefficients, where $N_{f}$ and $N_{b}$ are the number of forward and feedback taps respectively. Thus, the dimensions of the matrix depends on $L$. However, in our receiver structure, we solve matrix equations with the order of $N \times N$ for the LE where $N$ is total number of taps. And, for the DFE, we solve $N_{f} \times N_{f}$ matrix equations to calculate feedforward taps and calculate $N_{b}$ feedback taps by using forward taps and interpolated channel estimates [8, 1]. For example, Lo et al. chose $N_{f}=3$ and $N_{b}=2$. For the diversity order of $L=4$, they solved a $14 \times 14$ matrix equation. However, with the same number of taps (this number is also optimum for our system), we solve a $5 \times 5$ matrix equations for the LE or solve a $3 \times 3$ matrix equations and calculate 2 feedback taps for the DFE regardless of diversity. Hence, the computational complexity involved in calculation of the optimum diversity combiner and equalizer structure is considerably reduced as the number of diversity channels increases. In Figure 3, the average BER performance as a function of the normalized sampling rate, $\bar{f}$, for different values of the maximum Doppler frequency is illustrated for our ODCDFE( 3,2$)$ and Lo et al.'s receiver for the case of $S N R=20 \mathrm{~dB}$ and $L=1$. We see from the figure that ODCDFE(3,2) structure is slightly better than Lo et al.'s model for high maximum Doppler frequencies and the normalized sampling rate less than $2,(\bar{f}<2)$. For low Doppler frequencies and $\bar{f} \geq 2$, the receiver structures perform almost identically. We also observe similar effect as we increase the number of diversity channels [8]. The main reason for this improvement is the use of the fractionally spaced matched filter for each diversity branch. Since the matched filter spans the whole CIR, we maximize the power of the current symbol. 
The average BER performance as a function of the average channel SNR for maximum Doppler frequency, $f_{d m}$, is illustrated in Figure 4 for $L=1$ and Figure 5 for $L=2$. As expected, the DFE has much better performance than the LE since it is not sensitive to channel nulls. Although the system performance improves with increasing SNR, an irreducible BER was found to occur at high values of $S N R \geq 20 \mathrm{~dB}$. This is due to receiver limitations in estimation and tracking the rapid time variations in the fading channel. The use of diversity channels improves the system performance. Although it does not eliminate the error floor, it does reduce the level of the irreducible BER.

\section{Conclusions}

A simplified antenna diversity combiner-equalizer structure was proposed and analyzed for CMR channel. This receiver is preferable to previously proposed approaches when CIR estimation rather than adaptive equalization is performed. Further research issues include "phase alignment" problem [10] which arises in CIR estimation for differentially encoded data and receiver design for systems with error control coding.

\section{REFERENCES}

[1] J. G. Proakis, Digital Communications, McGraw-Hill, New York, 1995.

[2] S. Stein, "Fading Channel Issues in System Engineering", IEEE Journal on Selected Areas in Communications, 5(2):68-89, February 1987.

[3] N. W. K. Lo, D. D. Falconer, and A. U. H. Sheikh, "Adaptive Equalization and Diversity Combining for a Mobile Radio Channel using Interpolated Channel Estimates", IEEE Transactions on Vehicular Technology, 40(3):636-645, August 1991.

[4] P. Balaban and J. Salz, "Optimum Diversity Combining and Equalization in digital Data Transmission with Applications to Cellular Mobile Radio-Part I: Theoretical Considerations", IEEE Transactions on Communications, 40(5):885-894, May 1992.

[5] J. Weck, P. Merki and R. W. Lorenz, "Power Delay Profiles Measured in Mountainous Terrain", Proceedings of VTC, 105-112, September 1988.

[6] W. C. Jakes, Microwave Mobile Communications, John Wiley and Sons, New York, 1974.

[7] P. Dent, G. E. Bottomley, and T. Croft, "Jakes Fading Model Revisited", Electronics Letters, 29(13):1162-1163, June 1993.

[8] T. Eyceöz, "Reduced Complexity Optimal Diversity Combining and Adaptive Equalization Using Interpolated Channel Estimates with Applications to Cellular Mobile Radio Channels", Master's thesis, North Carolina State Univ., November 1995.

[9] T. Eyceöz and A. Duel-Hallen, "Reduced Complexity Diversity Combining and Adaptive Equalization Using Interpolated Channel Estimates with Applications to Cellular Mobile Radio Channels", Proceedings of ICUPC, 51-55 Sep. 30-Oct. 2, 1996.

[10] R. D. Koilpillai, S. Chennakeshu, and R. L. Toy, "Low Complexity Equalizers for U.S. Digital Cellular System", Proceedings of VTC, 744-747, May 1992. 


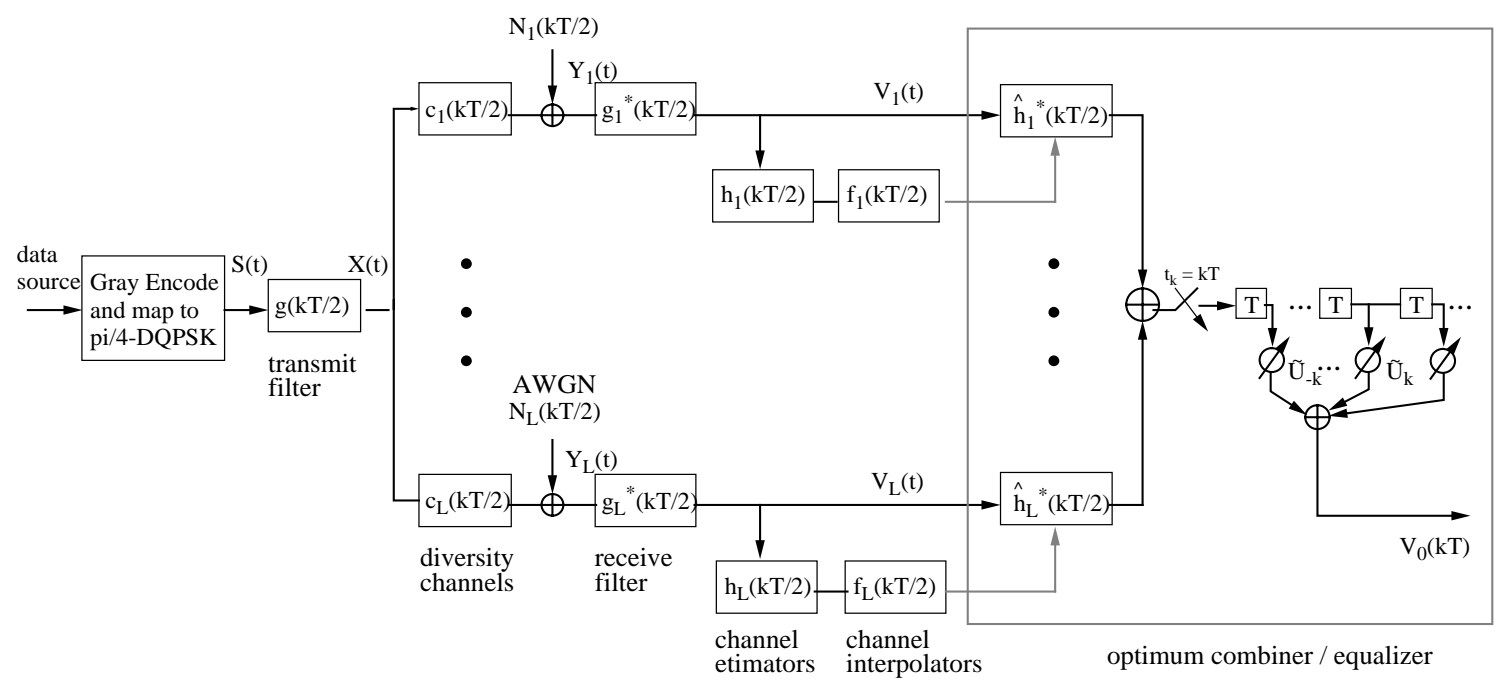

(a)

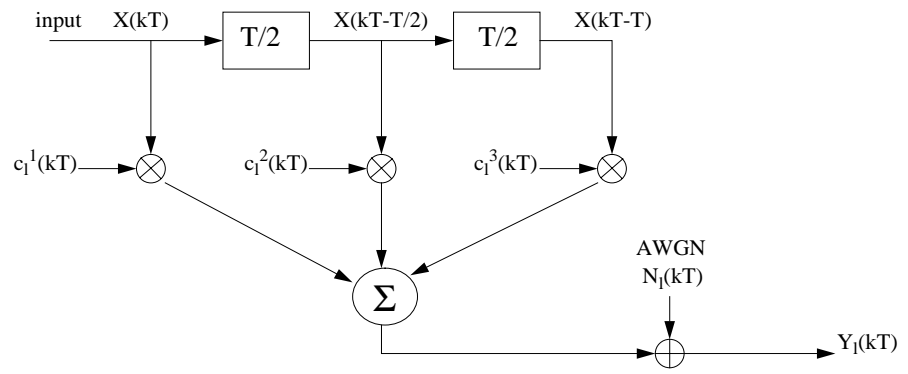

(b)



(c)

Figure 1: (a) CMR communications system with linear block adaptive equalization. (b) $T / 2$ spaced TDL with three-paths "mountainous terrain" channel model at $l^{\text {th }}$ diversity branch. (c) CIR interpolation within a TDMA time slot. 'o'-estimated CIR sample at the end of a training sequence, $\mathrm{Q}=4$, ' $\mathrm{x}$ '-interpolated CIR in the middle data frame, $R=T_{s} / T_{s}^{\prime}=4$ 


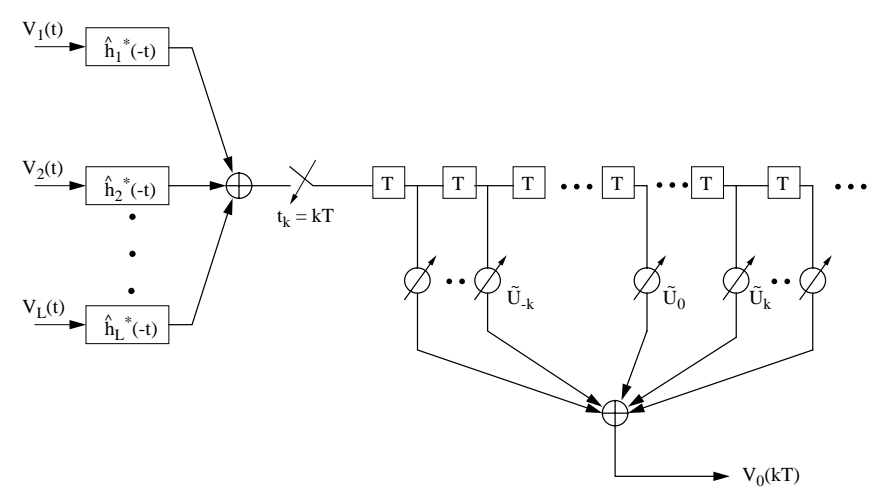

(a)

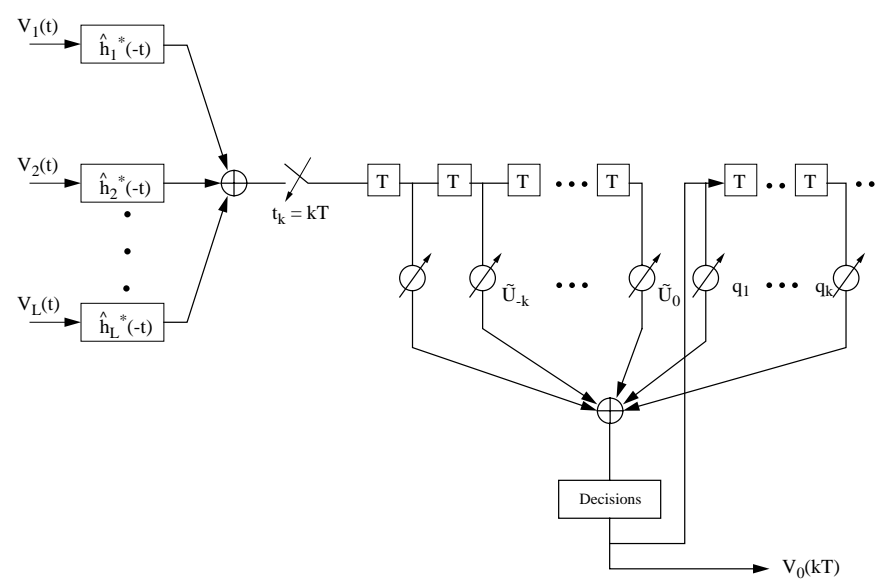

(b)

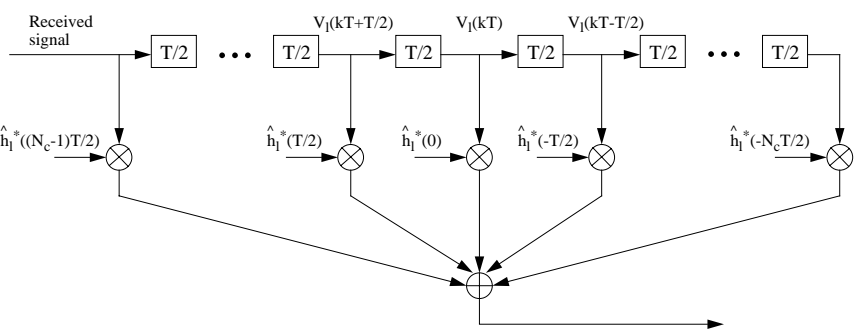

(c)

Figure 2: (a) Optimum diversity combiner linear equalizer structure. (b) Optimum diversity combiner decision feedback equalizer structure. (c) Fractionally spaced Matched Filter for the $l^{\text {th }}$ diversity branch. 




Figure 3: Performance comparison of our receiver structure and Lo et al.'s receiver structure as a function of the normalized sampling rate, $\bar{f}$, and the maximum Doppler frequency, $f_{d m}$ with $S N R=20 \mathrm{~dB}$ and $L=1$

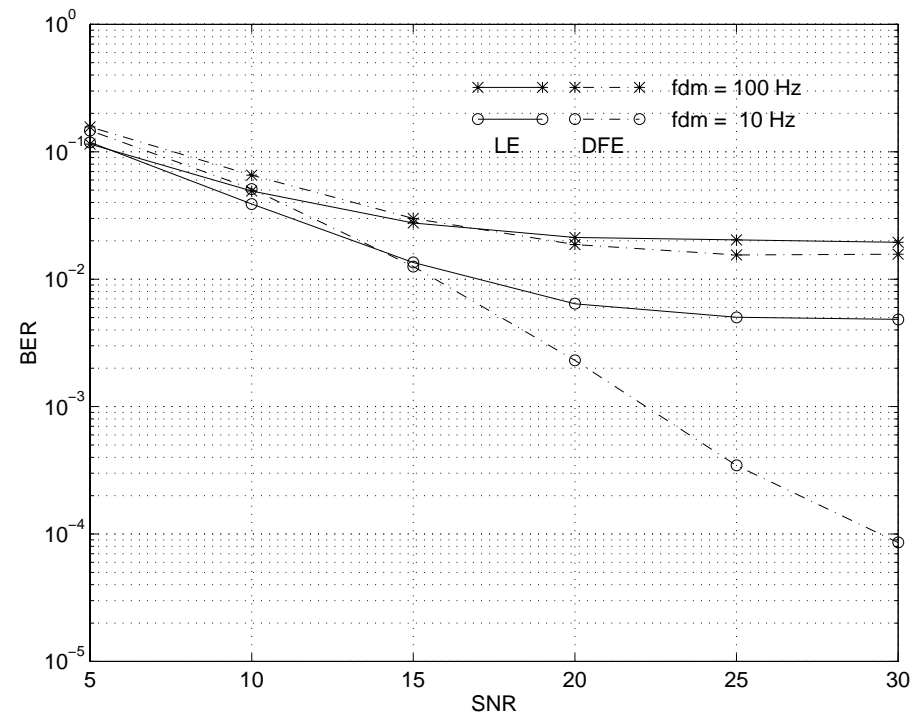

Figure 4: Average BER as a function of channel SNR, diversity and Doppler fading for $\operatorname{ODCLE}(5,0)$ and $\operatorname{ODCDFE}(3,2), \bar{f}=2.0$ and $L=1$ 


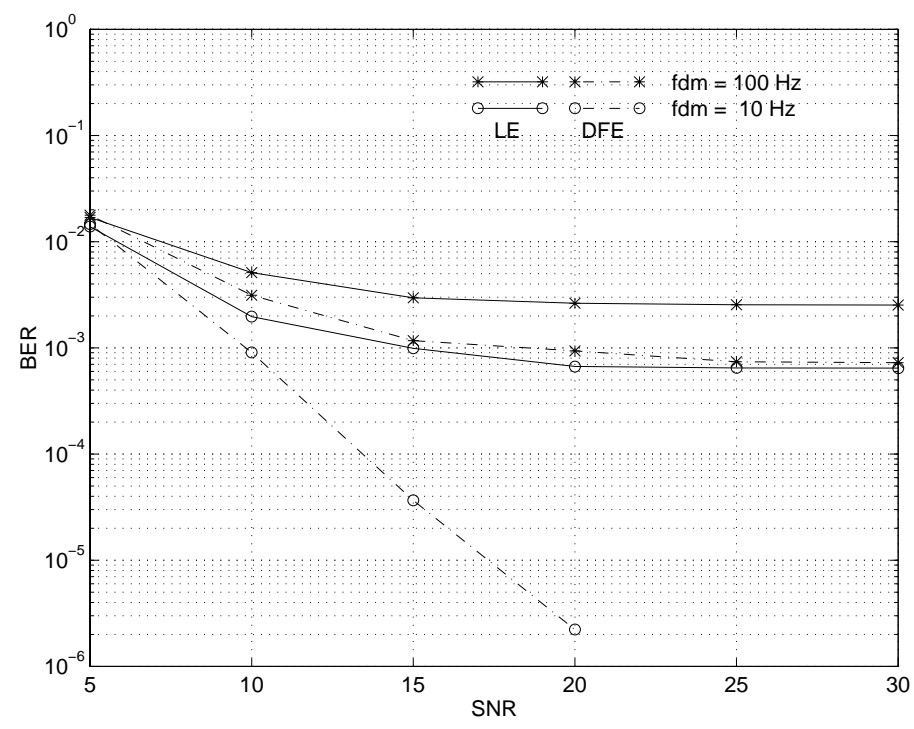

Figure 5: Average BER as a function of channel SNR, diversity and Doppler fading for $\operatorname{ODCLE}(5,0)$ and $\operatorname{ODCDFE}(3,2), \bar{f}=2.0$ and $L=2$ 